The Journal of Fourier Analysis and Applications

Volume 11, Issue 3, 2005

\title{
Generalized Shift-Invariant Systems and Frames for Subspaces
}

\author{
Ole Christensen and Yonina C. Eldar \\ Communicated by Dave Walnut
}

\begin{abstract}
Let $T_{k}$ denote translation by $k \in \mathbb{Z}^{d}$. Given countable collections of functions $\left\{\phi_{j}\right\}_{j \in J},\left\{\tilde{\phi_{j}}\right\}_{j \in J} \subset L^{2}\left(\mathbb{R}^{d}\right)$ and assuming that $\left\{T_{k} \phi_{j}\right\}_{j \in J, k \in \mathbb{Z}^{d}}$ and $\left\{T_{k} \tilde{\phi}_{j}\right\}_{j \in J, k \in \mathbb{Z}^{d}}$ are Bessel sequences, we are interested in expansions$$
f=\sum_{j \in J} \sum_{k \in \mathbb{Z}^{d}}\left\langle f, T_{k} \tilde{\phi}_{j}\right| T_{k} \phi_{j}, \forall f \in \overline{\operatorname{span}}\left\{T_{k} \phi_{j}\right\}_{k \in \mathbb{Z}^{d}, j \in J} .
$$

Our main result gives an equivalent condition for this to hold in a more general setting than described here, where translation by $k \in \mathbb{Z}^{d}$ is replaced by translation via the action of a matrix. As special cases of our result we find conditions for shift-invariant systems, Gabor systems, and wavelet systems to generate a subspace frame with a corresponding dual having the same structure.
\end{abstract}

\section{Introduction}

Given a real and invertible $d \times d$ matrix $C$, we define for $k \in \mathbb{Z}^{d}$ a generalized translation operator $T_{C k}$ acting on $f \in L^{2}\left(\mathbb{R}^{d}\right)$ by

$$
\left(T_{C k} f\right)(x)=f(x-C k), \quad x \in \mathbb{R}^{d} .
$$

A generalized shift-invariant system is a system of the type $\left\{T_{C_{j} k} \phi_{j}\right\}_{j \in J, k \in \mathbb{Z}^{d}}$, where $\left\{C_{j}\right\}_{j \in J}$ is a countable collection of real invertible $d \times d$ matrices, and $\left\{\phi_{j}\right\}_{j \in J} \subset L^{2}\left(\mathbb{R}^{d}\right)$. Generalized shift-invariant systems contain the classical wavelet systems and Gabor systems as special cases. Given the matrices $\left\{C_{j}\right\}_{j \in J}$, we are interested in functions $\left\{\phi_{j}\right\}_{j \in J}$, $\left\{\tilde{\phi}_{j}\right\}_{j \in J} \subset L^{2}\left(\mathbb{R}^{d}\right)$ for which $\left\{T_{C_{j} k} \phi_{j}\right\}_{j \in J, k \in \mathbb{Z}^{d}}$ and $\left\{T_{C_{j} k} \tilde{\phi}_{j}\right\}_{j \in J, k \in \mathbb{Z}^{d}}$ are Bessel sequences

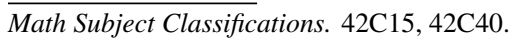

Keywords and Phrases. Frames for subspaces, generalized shift-invariant systems, wavelet systems, Gabor systems. 
and the expansions

$$
f=\sum_{j \in J} \sum_{k \in \mathbb{Z}^{d}}\left\langle f, T_{C_{j} k} \tilde{\phi}_{j}\right\rangle T_{C_{j} k} \phi_{j}, \forall f \in \overline{\operatorname{span}}\left\{T_{C_{j} k} \phi_{j}\right\}_{k \in \mathbb{Z}^{d}, j \in J}
$$

hold. Our main result, developed in Section 4, provides necessary and sufficient conditions such that the expansion (1.1) holds; they are based on an analysis of the case of a single generator, which is considered in Section 3.

Our conditions for (1.1) to hold are formulated in terms of the Fourier transform of the generators $\phi_{j}$ and $\tilde{\phi}_{j}$. For instance, letting $C_{j}^{\sharp}$ denote the inverse of the transpose of $C_{j}$, we prove that (1.1) holds, if and only if for all $\ell \in J, m \in \mathbb{Z}$ the equations

$$
\hat{\phi}_{\ell}(\gamma)=\sum_{j \in J} \frac{1}{\left|\operatorname{det} C_{j}\right|} \hat{\phi}_{j}(\gamma)\left(\sum_{n \in \mathbb{Z}^{d}} e^{-2 \pi i C_{\ell} m \cdot C_{j}^{\sharp} n} \hat{\phi}_{\ell}\left(\gamma+C_{j}^{\sharp} n\right) \overline{\hat{\tilde{\phi}}_{j}\left(\gamma+C_{j}^{\sharp} n\right)}\right)
$$

hold for a.e. $\gamma$. The parameters $\ell, m$ only appear in the study of frames for subspaces: In fact, assuming that $\left\{T_{C_{j} k} \phi_{j}\right\}_{j \in J, k \in \mathbb{Z}^{d}}$ is complete in $L^{2}\left(\mathbb{R}^{d}\right)$ (and a weak technical condition) Hernandez, Labate, and Weiss [11] have characterized (1.1), and the $\ell, m$ do not show up in this context. We discuss this issue after Corollary 1. We will also show that the condition (1.2) simplifies for some of the important systems considered in signal processing.

In Section 5 we consider the case of shift-invariant systems on $L^{2}(\mathbb{R})$, in which case the matrices $C_{j}$ correspond to multiplication with a constant $a$ : In this case, it is clear from (1.2) that the parameter $m$ can be eliminated from the general characterization. Section 6 specializes the results to the case of Gabor expansions; here, we can also eliminate the parameter $\ell$. Finally, in Section 7 we consider the case of a wavelet expansion. It turns out that our general result in Section 4 also simplifies in this case, but in a different way than in the shift-invariant case: The dependence on the parameter $\ell$ can be eliminated, but $m$ remains.

In the rest of this section we motivate our results by a short review of the present stage of frame theory.

A classical argument for frames is that they lead to expansions of the elements in the underlying Hilbert space in terms of the frame elements. To be more specific, if $\mathcal{H}$ is a separable Hilbert space and $\left\{f_{k}\right\}_{k=1}^{\infty}$ is a frame for $\mathcal{H}$ with frame operator $S$, then

$$
f=\sum_{k=1}^{\infty}\left\langle f, S^{-1} f_{k}\right\rangle f_{k}, \forall f \in \mathcal{H} .
$$

The family $\left\{S^{-1} f_{k}\right\}_{k=1}^{\infty}$ is called the canonical dual frame of $\left\{f_{k}\right\}_{k=1}^{\infty}$. In order to use the representation (1.3) in practice, we need to be able to calculate the coefficients $\left\langle f, S^{-1} f_{k}\right\rangle$. In general, this is highly nontrivial: While the existence of $S^{-1}$ is guaranteed by the frame condition, it is usually tedious to find this operator explicitly. The next best - approximation of $S^{-1}$, or at least $\left\langle f, S^{-1} f_{k}\right\rangle$-is also complicated. Another issue is that the frame $\left\{f_{k}\right\}_{k=1}^{\infty}$ might have a certain structure which is not shared by $\left\{S^{-1} f_{k}\right\}_{k=1}^{\infty}$. From a signal processing perspective, the missing structure of $\left\{S^{-1} f_{k}\right\}_{k=1}^{\infty}$ will make it complicated to deal with (1.3). This complication appears, e.g., if $\left\{f_{k}\right\}_{k=1}^{\infty}$ is a wavelet frame: In general, the canonical dual of a wavelet frame does not have the wavelet structure, cf. [5].

Finally, it might be difficult to control the properties of $\left\{S^{-1} f_{k}\right\}_{k=1}^{\infty}$; that is, if we want $\left\{S^{-1} f_{k}\right\}_{k=1}^{\infty}$ to have certain properties, we do in general not have a procedure which can tell us how to chose $\left\{f_{k}\right\}_{k=1}^{\infty}$ such that this is achieved. 
One way to circumvent these types of problems is to search for a pair of dual frames rather than just one frame: That is, we search for two frames $\left\{f_{k}\right\}_{k=1}^{\infty},\left\{g_{k}\right\}_{k=1}^{\infty}$ such that

$$
f=\sum_{k=1}^{\infty}\left\langle f, g_{k}\right\rangle f_{k}, \forall f \in \mathcal{H} .
$$

This approach opens for the possibility to specify in advance which properties, or structure we want for $\left\{f_{k}\right\}_{k=1}^{\infty}$ as well as $\left\{g_{k}\right\}_{k=1}^{\infty}$. For example, we can ask for both of them having wavelet structure. This is not merely theoretically possible: In fact, Bownik and Weber gave in [1] an example of a wavelet frame for which

(i) the canonical dual does not have wavelet structure;

(ii) infinitely many other duals with wavelet structure exist.

Our approach is in fact more general than described here: We also consider the case where (1.4) only holds on a subspace, the $f_{k}$ belong to that subspace, and without the assumption of $\left\{g_{k}\right\}_{k=1}^{\infty}$ being a frame.

Despite the fact that the canonical dual of a Gabor frame (or frame of translates) always has the same structure, the theory developed here is important for these cases as well. For example, for a frame of translates, we proved in [3] that among the "classical duals" (i.e., the duals belonging to the space spanned by the frame), the canonical dual is the only one which has the translation structure. On the other hand, in the same article, we demonstrate that the general approach presented here leads to several other duals having the translation structure. This flexibility is attained because we do not require the dual to belong to the same space. Similar cases exist for the Gabor case. For example, Gabardo and Han [9] have considered the Gabor system $\left\{e^{2 \pi i m x} \chi_{[0, a[}(x-n)\right\}_{m, n \in \mathbb{Z}}$ : For $\left.a \in\right] 0,1[$, it is an overcomplete frame for a closed subspace of $L^{2}(\mathbb{R})$. Thus, it has many duals, but Gabardo and Han prove that only the canonical dual has the Gabor structure.

The first step towards a general theory for dual frame pairs was given by Wexler and Raz [18]: Given a Gabor frame for $\ell^{2}(\mathbb{Z})$, their work characterized all duals having the Gabor structure. For shift-invariant systems $\left\{g_{m}(\cdot-n a)\right\}_{m, n \in \mathbb{Z}}$ in $L^{2}(\mathbb{R})$, the analogue problem was solved by Janssen [13] and, independently, Ron and Shen [15]. For wavelet systems, the answer appeared in [8] by Frazier et al.

More recently, several authors have considered generalized shift-invariant systems, which contain Gabor systems, general shift-invariant systems, and wavelet systems as special cases. Characterizations of dual frame pairs in this setting have been obtained by Hernandez, Labate, and Weiss [11] as well as by Ron and Shen [16].

All the results discussed so far concern the case where we are interested in a frame decomposition which is valid for all elements in the underlying Hilbert space. The results in this article are more general: In fact, our results concern representations of the type (1.4) for all $f$ belonging to a closed subspace $X$ of $L^{2}(\mathbb{R})$, with the additional assumption that $\left\{f_{k}\right\}$ belongs to $X$.

We note that Li and Ogawa [14] have introduced pseudoframe decompositions for closed subspaces $X$ of general Hilbert spaces: Here, (1.4) is required to hold only for $f \in X$, but without assuming that $\left\{f_{k}\right\}_{k=1}^{\infty}$ and $\left\{g_{k}\right\}_{k=1}^{\infty}$ are frames for $X$. In fact, they are allowed not to belong to $X$. Thus, pseudoframes are more general than our approach; it is an interesting open problem how one can extend our results to the setting of pseudo-frames.

We also note that in $[6,7,17,3]$, the authors consider oblique frame decompositions in which, as for pseudoframes, (1.4) is required to hold only for $f \in X$. In these articles $\left\{f_{k}\right\}_{k=1}^{\infty}$ forms a frame, and the vectors $\left\{g_{k}\right\}_{k=1}^{\infty}$ also form a frame but for a different subspace $Y$ such that $\mathcal{H}=X \oplus Y^{\perp}$. 
We present our results in the same generality as the above mentioned results by Hernandez et al. and Ron and Shen. Under a technical condition, a corollary of our main result recovers the main statement about generalized shift invariant systems in [11] as a special case.

\section{Notation and Preliminary Results}

As stated already in the introduction we consider generalized shift-invariant systems of the type $\left\{T_{C_{j} k} \phi_{j}\right\}_{j \in J, k \in \mathbb{Z}^{d}}$, where $\left\{C_{j}\right\}_{j \in J}$ is a countable collection of real invertible $d \times d$ matrices, and $\left\{\phi_{j}\right\}_{j \in J} \subset L^{2}\left(\mathbb{R}^{d}\right)$. Letting $C^{T}$ denote the transpose of an invertible matrix $C$, we use the notation

$$
C^{\sharp}=\left(C^{T}\right)^{-1}
$$

For $f \in L^{1}\left(\mathbb{R}^{d}\right)$ we denote the Fourier transform by

$$
\mathcal{F} f(\gamma)=\hat{f}(\gamma)=\int_{\mathbb{R}^{d}} f(x) e^{-2 \pi i x \cdot \gamma} d x,
$$

where $x \cdot \gamma$ denotes the inner product between $x$ and $\gamma$. As usual, the Fourier transform is extended to a unitary operator on $L^{2}\left(\mathbb{R}^{d}\right)$. Furthermore,

$$
\mathcal{F} T_{C k} f(\gamma)=e^{-2 \pi i C k \cdot \gamma} \hat{f}(\gamma)
$$

With $E_{b}(x):=e^{2 \pi i b \cdot x}, b, x \in \mathbb{R}^{d}$, this yields the commutator relation

$$
\mathcal{F} T_{C k}=E_{-C k} \mathcal{F}
$$

While we present the general theory for the $d$-dimensional case, our discussion of shift-invariant systems, Gabor systems, and wavelets will take place in $L^{2}(\mathbb{R})$. Generalized shift-invariant systems in $L^{2}(\mathbb{R})$ will be denoted by

$$
\left\{T_{a_{j} k} \phi_{j}\right\}_{j \in J, k \in \mathbb{Z}}, \quad \text { where } a_{j}>0, \phi_{j} \in L^{2}(\mathbb{R})
$$

Most of our calculations rely on Fourier transformation techniques rather than general Hilbert space results. However, we need a lemma which is most conveniently formulated for general Hilbert spaces:

Lemma 1. Let $\left\{f_{k}\right\}_{k=1}^{\infty}$ and $\left\{g_{k}\right\}_{k=1}^{\infty}$ be Bessel sequences in a Hilbert space $\mathcal{H}$, and assume that

$$
f_{j}=\sum_{k=1}^{\infty}\left\langle f_{j}, g_{k}\right\rangle f_{k}, \forall j \in \mathbb{N}
$$

Then $\left\{f_{k}\right\}_{k=1}^{\infty}$ is a frame for $\overline{\operatorname{span}}\left\{f_{k}\right\}_{k=1}^{\infty}$, and

$$
f=\sum_{k=1}^{\infty}\left\langle f, g_{k}\right\rangle f_{k}, \forall f \in \overline{\operatorname{span}}\left\{f_{k}\right\}_{k=1}^{\infty} \text {. }
$$


Proof. The assumptions immediately imply that (2.2) holds for $f \in \operatorname{span}\left\{f_{k}\right\}_{k=1}^{\infty}$. By the Bessel assumption, the operator $f \mapsto \sum_{k=1}^{\infty}\left\langle f, g_{k}\right\rangle f_{k}$ is continuous, and therefore (2.2) actually holds for all $f \in \overline{\text { span }}\left\{f_{k}\right\}_{k=1}^{\infty}$. Finally, Cauchy-Schwarz' inequality applied to

$$
\|f\|^{2}=\sum_{k=1}^{\infty}\left\langle f, g_{k}\right\rangle\left\langle f_{k}, f\right\rangle, f \in \overline{\operatorname{span}}\left\{f_{k}\right\}_{k=1}^{\infty},
$$

yields the announced frame property.

Remark. The assumption about $\left\{g_{k}\right\}_{k=1}^{\infty}$ being a Bessel sequence can not be removed from Lemma 1. See Example 5.4.6 in [2].

A sequence $\left\{f_{k}\right\}_{k=1}^{\infty}$ in $\mathcal{H}$ which is a frame for $\overline{\operatorname{span}}\left\{f_{k}\right\}_{k=1}^{\infty}$ is called a frame sequence. Given a frame sequence $\left\{f_{k}\right\}_{k=1}^{\infty}$, any Bessel sequence $\left\{g_{k}\right\}_{k=1}^{\infty}$ in $\mathcal{H}$ for which

$$
f=\sum_{k=1}^{\infty}\left\langle f, g_{k}\right\rangle f_{k}, \forall f \in \overline{\operatorname{span}}\left\{f_{k}\right\}_{k=1}^{\infty}
$$

will be called a generalized dual of $\left\{f_{k}\right\}_{k=1}^{\infty}$.

\section{The Case of One Generator}

In this section we consider translates (in the general sense stated in Section 1) of a single function. The results obtained here will serve as starting point for the case of multiple generators.

Lemma 2. Let $\phi, \tilde{\phi} \in L^{2}\left(\mathbb{R}^{d}\right)$, let $C$ be a real and invertible $d \times d$ matrix, and assume that $\left\{T_{C k} \phi\right\}_{k \in \mathbb{Z}^{d}}$ and $\left\{T_{C k} \tilde{\phi}\right\}_{k \in \mathbb{Z}^{d}}$ are Bessel sequences. Then for all $f \in L^{2}\left(\mathbb{R}^{d}\right)$,

$$
\mathcal{F}\left(\sum_{k \in \mathbb{Z}^{d}}\left\langle f, T_{C k} \tilde{\phi}\right| T_{C k} \phi\right)=\frac{1}{|\operatorname{det} C|} \hat{\phi}(\gamma) \sum_{n \in \mathbb{Z}^{d}} \hat{f}\left(\gamma+C^{\sharp} n\right) \overline{\tilde{\hat{\phi}}\left(\gamma+C^{\sharp} n\right)} .
$$

Proof. First, observe that

$$
\begin{aligned}
& \left\langle f, T_{C k} \tilde{\phi}\right\rangle=\int_{\mathbb{R}^{d}} \hat{f}(\mu) \overline{\tilde{\tilde{\phi}}(\mu)} e^{2 \pi i C k \cdot \mu} d \mu \\
& =\int_{\mathbb{R}^{d}} \hat{f}(\mu) \overline{\tilde{\tilde{\phi}}(\mu)} e^{2 \pi i k \cdot C^{T} \mu} d \mu \\
& =\frac{1}{\left|\operatorname{det} C^{T}\right|} \int_{\mathbb{R}^{d}} \hat{f}\left(C^{\sharp} \mu\right) \overline{\hat{\tilde{\phi}}\left(C^{\sharp} \mu\right)} e^{2 \pi i k \cdot \mu} d \mu \\
& =\frac{1}{|\operatorname{det} C|} \int_{\mathbb{T}^{d}} \sum_{n \in \mathbb{Z}^{d}} \hat{f}\left(C^{\sharp}(\mu+n)\right) \overline{\hat{\tilde{\phi}}\left(C^{\sharp}(\mu+n)\right)} e^{2 \pi i k \cdot \mu} d \mu \text {. }
\end{aligned}
$$

Here we used the notation $\mathbb{T}=[0,1]$. It follows that 


$$
\begin{aligned}
\mathcal{F} & \left(\sum_{k \in \mathbb{Z}^{d}}\left\langle f, T_{C k} \tilde{\phi}\right| T_{C k} \phi(\gamma)\right) \\
= & \sum_{k \in \mathbb{Z}^{d}}\left\langle f, T_{C k} \tilde{\phi}\right| \mathcal{F} T_{C k} \phi(\gamma) \\
= & \frac{1}{|\operatorname{det} C|} \sum_{k \in \mathbb{Z}^{d}}\left(\int_{\mathbb{T}^{d}} \sum_{n \in \mathbb{Z}^{d}} \hat{f}\left(C^{\sharp}(\mu+n)\right) \overline{\hat{\tilde{\phi}}\left(C^{\sharp}(\mu+n)\right)} e^{2 \pi i k \cdot \mu} d \mu\right) \\
= & \frac{1}{|\operatorname{det} C|} \hat{\phi}(\gamma) \sum_{n \in \mathbb{Z}^{d}} \hat{f}\left(C^{\sharp}\left(C^{T} \gamma+n\right)\right) \overline{\hat{\tilde{\phi}}\left(C^{\sharp}\left(C^{T} \gamma+n\right)\right)} \\
= & \frac{1}{|\operatorname{det} C|} \hat{\phi}(\gamma) \sum_{n \in \mathbb{Z}^{d}} \hat{f}\left(\gamma+C^{\sharp} n\right) \overline{\hat{\tilde{\phi}}\left(\gamma+C^{\sharp} n\right)} .
\end{aligned}
$$

Lemma 2 immediately leads to a characterization of the desired type for systems generated by a single function. It extends a result from [3] to the multivariable setting, with the general matrix translation considered here:

Proposition 1. Let $C$ be a real and invertible $d \times d$ matrix and $\phi, \tilde{\phi} \in L^{2}\left(\mathbb{R}^{d}\right)$. Assume that $\left\{T_{C k} \phi\right\}_{k \in \mathbb{Z}^{d}}$ and $\left\{T_{C k} \tilde{\phi}\right\}_{k \in \mathbb{Z}^{d}}$ are Bessel sequences. Then the following are equivalent:

(i) $f=\sum_{k \in \mathbb{Z}^{d}}\left\langle f, T_{C k} \tilde{\phi}\right\rangle T_{C k} \phi, \forall f \in \overline{\operatorname{span}}\left\{T_{C k} \phi\right\}_{k \in \mathbb{Z}^{d}}$.

(ii) $\operatorname{On}\left\{\gamma: \sum_{n \in \mathbb{Z}^{d}}\left|\hat{\phi}\left(\gamma+C^{\sharp} n\right)\right|^{2} \neq 0\right\}$, we have

$$
\sum_{n \in \mathbb{Z}^{d}} \hat{\phi}\left(\gamma+C^{\sharp} n\right) \overline{\tilde{\tilde{\phi}}\left(\gamma+C^{\sharp} n\right)}=|\operatorname{det} C| \text {, a.e. }
$$

If the conditions are satisfied, then $\left\{T_{C k} \phi\right\}_{k \in \mathbb{Z}^{d}}$ is a frame for $\overline{\operatorname{span}}\left\{T_{C k} \phi\right\}_{k \in \mathbb{Z}^{d}}$; furthermore, the orthogonal projection of $\left\{T_{C k} \tilde{\phi}\right\}_{k \in \mathbb{Z}^{d}}$ onto span $\left\{T_{C k} \phi\right\}_{k \in \mathbb{Z}^{d}}$ is a frame for $\overline{\operatorname{span}}\left\{T_{C k} \phi\right\}_{k \in \mathbb{Z}^{d}}$.

Proof. Assume that (i) holds. Via Lemma 2 with $f=\phi$,

$$
\hat{\phi}(\gamma)=\frac{1}{|\operatorname{det} C|} \hat{\phi}(\gamma) \sum_{n \in \mathbb{Z}^{d}} \hat{\phi}\left(\gamma+C^{\sharp} n\right) \overline{\hat{\tilde{\phi}}\left(\gamma+C^{\sharp} n\right)} .
$$

Replacing $\gamma$ with $\gamma+C^{\sharp} k$ shows that for all $k \in \mathbb{Z}^{d}$,

$$
\hat{\phi}\left(\gamma+C^{\sharp} k\right)=\frac{1}{|\operatorname{det} C|} \hat{\phi}\left(\gamma+C^{\sharp} k\right) \sum_{n \in \mathbb{Z}^{d}} \hat{\phi}\left(\gamma+C^{\sharp} n\right) \overline{\hat{\tilde{\phi}}\left(\gamma+C^{\sharp} n\right)} .
$$

This proves (ii). On the other hand, if (ii) holds then Lemma 2 shows that for any $k^{\prime} \in \mathbb{Z}^{d}$, 


$$
\begin{aligned}
\mathcal{F} & \sum_{k \in \mathbb{Z}^{d}}\left\langle T_{C k^{\prime}} \phi, T_{C k} \tilde{\phi}\right| T_{C k} \phi(\gamma) \\
& =\frac{1}{|\operatorname{det} C|} \hat{\phi}(\gamma) \sum_{n \in \mathbb{Z}^{d}} \mathcal{F} T_{C k^{\prime}} \phi\left(\gamma+C^{\sharp} n\right) \overline{\hat{\tilde{\phi}}\left(\gamma+C^{\sharp} n\right)} \\
& =\frac{1}{|\operatorname{det} C|} \hat{\phi}(\gamma) \sum_{n \in \mathbb{Z}^{d}} e^{-2 \pi i C k^{\prime} \cdot\left(\gamma+C^{\sharp} n\right)} \hat{\phi}\left(\gamma+C^{\sharp} n\right) \overline{\tilde{\tilde{\phi}}\left(\gamma+C^{\sharp} n\right)} \\
& =\frac{1}{|\operatorname{det} C|} \sum_{n \in \mathbb{Z}^{d}} \hat{\phi}\left(\gamma+C^{\sharp} n\right) \overline{\tilde{\tilde{\phi}}\left(\gamma+C^{\sharp} n\right)} e^{-2 \pi i C k^{\prime} \cdot \gamma} \hat{\phi}(\gamma) \\
& =\mathcal{F} T_{C k^{\prime}} \phi(\gamma) .
\end{aligned}
$$

Thus,

$$
T_{C k^{\prime}} \phi=\sum_{k \in \mathbb{Z}^{d}}\left\langle T_{C k^{\prime}} \phi, T_{C k} \tilde{\phi}\right\rangle T_{C k} \phi .
$$

Now (i) follows via Lemma 1 . The rest is standard.

\section{Multiple Generators}

We now turn to the setting of multiple generators. Given a sequence of functions $\left\{\phi_{j}\right\}_{j \in J}$ in $L^{2}\left(\mathbb{R}^{d}\right)$ and a corresponding collection $\left\{C_{j}\right\}_{j \in J}$ of real and invertible $d \times d$ matrices, let

$$
\mathcal{W}=\overline{\operatorname{span}}\left\{T_{C_{j} k} \phi_{j}\right\}_{k \in \mathbb{Z}^{d}, j \in J},
$$

and let $P_{\mathcal{W}}$ denote the orthogonal projection onto $\mathcal{W}$.

Our first result below characterizes pairs of a frame $\left\{T_{C_{j} k} \phi_{j}\right\}_{k \in \mathbb{Z}^{d}, j \in J}$ and corresponding generalized duals $\left\{T_{C_{j} k} \tilde{\phi}_{j}\right\}_{k \in \mathbb{Z}^{d}, j \in J}$. The general classification is quite involved; the next sections show how it simplifies in concrete cases.

Theorem 1. Let $J$ be a countable index set and consider sequences $\left\{\phi_{j}\right\}_{j \in J}$ and $\left\{\tilde{\phi}_{j}\right\}_{j \in J}$ in $L^{2}\left(\mathbb{R}^{d}\right)$. Let $\left\{C_{j}\right\}_{j \in J}$ be a sequence of invertible real matrices, and assume that $\left\{T_{C_{j} k} \phi_{j}\right\}_{k \in \mathbb{Z}^{d}, j \in J}$ and $\left\{T_{C_{j} k} \tilde{\phi}_{j}\right\}_{k \in \mathbb{Z}^{d}, j \in J}$ are Bessel sequences. Then the following are equivalent:

(i) $f=\sum_{j \in J} \sum_{k \in \mathbb{Z}^{d}}\left\langle f, T_{C_{j} k} \tilde{\phi}_{j}\right\rangle T_{C_{j} k} \phi_{j}, \forall f \in \mathcal{W}$.

(ii) For all $\ell \in J, m \in \mathbb{Z}^{d}$,

$$
\hat{\phi}_{\ell}(\gamma)=\sum_{j \in J} \frac{1}{\left|\operatorname{det} C_{j}\right|} \hat{\phi}_{j}(\gamma)\left(\sum_{n \in \mathbb{Z}^{d}} e^{-2 \pi i C_{\ell} m \cdot C_{j}^{\sharp} n} \hat{\phi}_{\ell}\left(\gamma+C_{j}^{\sharp} n\right) \overline{\hat{\tilde{\phi}}_{j}\left(\gamma+C_{j}^{\sharp} n\right)}\right),
$$

holds for a.e. $\gamma$. If the conditions are satisfied, then $\left\{T_{C_{j} k} \phi_{j}\right\}_{k \in \mathbb{Z}^{d}, j \in J}$ and $\left\{P_{\mathcal{W}} T_{C_{j} k} \tilde{\phi}_{j}\right\}_{k \in \mathbb{Z}^{d}, j \in J}$ are dual frames for $\overline{\text { span }}\left\{T_{C_{j} k} \phi_{j}\right\}_{k \in \mathbb{Z}^{d}, j \in J}$.

Proof. Assume that (i) holds. Letting $f=T_{C_{\ell} m} \phi_{\ell}, m \in \mathbb{Z}^{d}, \ell \in J$, Lemma 2 shows that

$$
\mathcal{F} T_{C_{\ell} m} \phi_{\ell}(\gamma)=\sum_{j \in J} \frac{1}{\left|\operatorname{det} C_{j}\right|} \hat{\phi}_{j}(\gamma) \sum_{n \in \mathbb{Z}^{d}} \mathcal{F} T_{C_{\ell} m} \phi_{\ell}\left(\gamma+C_{j}^{\sharp} n\right) \overline{\hat{\tilde{\phi}}_{j}\left(\gamma+C_{j}^{\sharp} n\right)} ;
$$


since $\mathcal{F} T_{C_{\ell} m} \phi_{\ell}(\gamma)=e^{-2 \pi i C_{\ell} m \cdot \gamma} \hat{\phi}_{\ell}(\gamma)$ this proves (ii). On the other hand, if (ii) holds, then (i) holds for all $T_{C_{\ell} m} \phi_{\ell}, m \in \mathbb{Z}^{d}, \ell \in J$; via Lemma 1 this proves (i).

In the rest of this section we consider a reformulation of condition (ii) in Theorem 1. We note that in the summation over $n \in \mathbb{Z}^{d}$, the indices $j, n$ always appear in the combination $C_{j}^{\sharp} n$; let us consider all possible outcomes, i.e., let

$$
\Lambda=\left\{C_{j}^{\sharp} n: j \in J, n \in \mathbb{Z}^{d}\right\} .
$$

Given $\alpha \in \Lambda$, there might exist several pairs $(j, n) \in J \times \mathbb{Z}^{d}$ for which $\alpha=C_{j}^{\sharp} n$; let

$$
J_{\alpha}=\left\{j \in J: \exists n \in \mathbb{Z}^{d} \text { such that } \alpha=C_{j}^{\sharp} n\right\} .
$$

If we are allowed to reorder the sum in condition (ii) of Theorem 1, we can rewrite the condition in terms of the index sets $\Lambda$ and $J_{\alpha}$ :

Corollary 1. Assume the setup from Theorem 1 and define $\Lambda$ and $J_{\alpha}$ as in (4.1) and (4.2). Furthermore, assume that the series

$$
\sum_{\alpha \in \Lambda} \sum_{j \in J_{\alpha}} \frac{1}{\left|\operatorname{det} C_{j}\right|} \hat{\phi}_{j}(\gamma) e^{-2 \pi i C_{\ell} m \cdot \alpha} \hat{\phi}_{\ell}(\gamma+\alpha) \overline{\hat{\tilde{\phi}}_{j}(\gamma+\alpha)} \text {, a.e. } \gamma
$$

converges unconditionally for all $\ell \in J, m \in \mathbb{Z}$. Then the following are equivalent:

(i) $f=\sum_{j \in J} \sum_{k \in \mathbb{Z}^{d}}\left\langle f, T_{C_{j} k} \tilde{\phi}_{j}\right\rangle T_{C_{j} k} \phi_{j}, \forall f \in \mathcal{W}$.

(ii) For all $\ell \in J$ and all $m \in \mathbb{Z}^{d}$,

$$
\hat{\phi}_{\ell}(\gamma)=\sum_{\alpha \in \Lambda}\left(\sum_{j \in J_{\alpha}} \frac{1}{\left|\operatorname{det} C_{j}\right|} \hat{\phi}_{j}(\gamma) \overline{\tilde{\hat{\phi}}_{j}(\gamma+\alpha)}\right) e^{-2 \pi i C_{\ell} m \cdot \alpha} \hat{\phi}_{\ell}(\gamma+\alpha) \text {, a.e. } \gamma \text {. }
$$

In particular, the conditions (i) and (ii) are satisfied, if for all $\alpha \in \Lambda$,

$$
\sum_{j \in J_{\alpha}} \frac{1}{\left|\operatorname{det} C_{j}\right|} \hat{\phi_{j}}(\gamma) \overline{\tilde{\hat{\phi}}_{j}(\gamma+\alpha)}=\delta_{\alpha, 0} \text { a.e. on }\left\{\gamma: \sum_{j \in J}\left|\hat{\phi}_{j}(\gamma)\right|^{2} \neq 0\right\} \text {. }
$$

Proof. The series in (4.3) is a reordering of the series on the right-hand side of (ii) in Theorem 1; this leads to the first part of the conclusion. We now prove that (4.4) holds for all $\ell \in J$ and all $m \in \mathbb{Z}^{d}$, if (4.5) holds; in fact, since $J_{0}=J$,

$$
\begin{aligned}
& \sum_{\alpha \in \Lambda}\left(\sum_{j \in J_{\alpha}} \frac{1}{\left|\operatorname{det} C_{j}\right|} \hat{\phi}_{j}(\gamma) \overline{\hat{\tilde{\phi}}_{j}(\gamma+\alpha)}\right) e^{-2 \pi i C_{\ell} m \cdot \alpha} \hat{\phi}_{\ell}(\gamma+\alpha) \\
& =\left\{\begin{array}{lll}
\hat{\phi}_{\ell}(\gamma) & \text { if } & \sum_{j \in J}\left|\hat{\phi}_{j}(\gamma)\right|^{2} \neq 0 \\
0 & \text { if } & \sum_{j \in J}\left|\hat{\phi}_{j}(\gamma)\right|^{2}=0
\end{array}\right. \\
& =\hat{\phi}_{\ell}(\gamma) .
\end{aligned}
$$

We will return to the condition of unconditional convergence of (4.3) in the concrete cases of shift-invariant systems and Gabor systems in Section 5 and Section 6, respectively. 
Condition (4.4) exhibits $\hat{\phi}_{\ell}$ as a "generalized linear combination" of shifted versions of $\hat{\phi_{\ell}}(\cdot+\alpha), \alpha \in \Lambda$, where the word "generalized" refer to the coefficients depending on the variable $\gamma$. Condition (4.5) is trivially satisfied for all $\ell \in J$ and all $m \in \mathbb{Z}^{d}$, if

$$
\sum_{j \in J_{\alpha}} \frac{1}{\left|\operatorname{det} C_{j}\right|} \hat{\phi}_{j}(\gamma) \overline{\hat{\tilde{\phi}}_{j}(\gamma+\alpha)}=\delta_{\alpha, 0}, \quad \forall \alpha \in \Lambda
$$

Under a weak technical assumption, condition (4.6) is in [11] proved to be equivalent to $\left\{T_{C_{j} k} \phi_{j}\right\}_{k \in \mathbb{Z}^{d}, j \in J}$ and $\left\{T_{C_{j} k} \tilde{\phi}_{j}\right\}_{k \in \mathbb{Z}^{d}, j \in J}$ being dual frames for $L^{2}\left(\mathbb{R}^{d}\right)$; thus, modulo the issue of unconditional convergence, our general approach has recovered this result as a special case, except that we have not proved that $\left\{T_{C_{j} k} \phi_{j}\right\}_{k \in \mathbb{Z}^{d}, j \in J}$ is complete in $L^{2}\left(\mathbb{R}^{d}\right)$ in this special case.

Motivated by this classical result concerning frames for $L^{2}(\mathbb{R})$, one could ask whether the condition (4.5) is equivalent with (4.4). This turns out not to be the case. We will demonstrate this via an example in the context of shift-invariant systems - see Example 1. Comparison of our work with [11] yields a further conclusion:

Corollary 2. Assume the setup of Corollary 1. If the condition (4.4) is satisfied, but (4.6) is not, then $\left\{T_{C_{j} k} \phi_{j}\right\}_{k \in \mathbb{Z}^{d}, j \in J}$ is a frame for a proper subspace of $L^{2}\left(\mathbb{R}^{d}\right)$.

Remark. If $J$ is finite, then $\left\{T_{C_{j} k} \phi_{j}\right\}_{k \in \mathbb{Z}^{d}, j \in J}$ can not be a frame for $L^{2}\left(\mathbb{R}^{d}\right)$, cf. [4]. That is, (4.6) can not hold. But (4.4) can very well be satisfied.

\section{Shift-Invariant Systems}

Let us now consider shift-invariant systems on $L^{2}(\mathbb{R})$. With an adoption of our notation in (2.1) we write such a system as $\left\{T_{k a} \phi_{j}\right\}_{k \in \mathbb{Z}, j \in J}$, where $a>0$. As a special case of Corollary 1 we have

Corollary 3. Let $J$ be a countable index set and $\left\{\phi_{j}\right\}_{j \in J},\left\{\tilde{\phi}_{j}\right\}_{j \in J} \subset L^{2}(\mathbb{R})$. Let a $>0$, assume that $\left\{T_{k a} \phi_{j}\right\}_{k \in \mathbb{Z}, j \in J}$ and $\left\{T_{k a} \tilde{\phi}_{j}\right\}_{k \in \mathbb{Z}, j \in J}$ are Bessel sequences, and that the series

$$
\sum_{j \in J} \sum_{n \in \mathbb{Z}} \hat{\phi}_{j}(\gamma) \hat{\phi}_{\ell}(\gamma+n / a) \overline{\hat{\tilde{\phi}}_{j}(\gamma+n / a)}
$$

converges unconditionally for all $\ell \in J$. Then the following are equivalent:

(i) $f=\sum_{j \in J} \sum_{k \in \mathbb{Z}}\left\langle f, T_{k a} \tilde{\phi}_{j}\right\rangle T_{k a} \phi_{j}, \forall f \in \overline{\operatorname{span}}\left\{T_{k a} \phi_{j}\right\}_{k \in \mathbb{Z}, j \in J}$.

(ii) For all $\ell \in J$,

$$
\hat{\phi}_{\ell}(\gamma)=\sum_{n \in \mathbb{Z}}\left(\frac{1}{a} \sum_{j \in J} \hat{\phi}_{j}(\gamma) \overline{\hat{\tilde{\phi}}_{j}(\gamma+n / a)}\right) \hat{\phi}_{\ell}(\gamma+n / a), \text { a.e. } \gamma \text {. }
$$

Condition (ii) holds in particular, if

$$
\sum_{j \in J} \hat{\phi}_{j}(\gamma) \overline{\tilde{\phi}_{j}(\gamma+n / a)}=a \delta_{n, 0} \text { a.e. on }\left\{\gamma: \sum_{j \in J}\left|\hat{\phi}_{j}(\gamma)\right|^{2} \neq 0\right\} \text {. }
$$


Note, in particular that the parameter $m$ appearing in Corollary 1 is eliminated in the condition (5.2). As we will see later, this is different from what happens in the wavelet case. We also note that it was proved by Janssen (see $[12,13]$, or [2], Therorem 8.7.2) that two pairs of Bessel sequences $\left\{T_{k a} \phi_{j}\right\}_{k \in \mathbb{Z}, j \in J}$ and $\left\{T_{k a} \tilde{\phi}_{j}\right\}_{k \in \mathbb{Z}, j \in J}$ are dual frames for $L^{2}(\mathbb{R})$, if and only if

$$
\frac{1}{a} \sum_{j \in J} \hat{\phi}_{j}(\gamma) \overline{\hat{\tilde{\phi}}_{j}(\gamma+n / a)}=\delta_{n, 0}
$$

thus, whether the condition (5.3) leads to $\left\{T_{k a} \phi_{j}\right\}_{k \in \mathbb{Z}, j \in J}$ being a frame for all of $L^{2}(\mathbb{R})$, or just for a subspace only depends on the set

$$
\left\{\gamma: \sum_{j \in J}\left|\hat{\phi}_{j}(\gamma)\right|^{2} \neq 0\right\}
$$

being empty, or not.

So far, we have not discussed the unconditional convergence of, e.g., (5.1). Below we present a condition which makes (5.1) converge unconditionally.

Lemma 3. Assume that $\left\{T_{k a} \phi_{j}\right\}_{k \in \mathbb{Z}, j \in J}$ and $\left\{T_{k a} \tilde{\phi}_{j}\right\}_{k \in \mathbb{Z}, j \in J}$ are Bessel sequences, and that

$$
\sum_{j \in J}\left|\hat{\phi}_{j}(\gamma)\right|<\infty \text {, a.e. }
$$

Then the series in (5.1) converges unconditionally for a.e. $\gamma$.

Proof. If $B$ denotes a bound for the Bessel sequence $\left\{T_{k a} \phi_{j}\right\}_{k \in \mathbb{Z}, j \in J}$, then $B$ is also a bound for $\left\{T_{k a} \phi_{j}\right\}_{k \in \mathbb{Z}}$ for any $j \in J$; it is well known that this implies that, independently of $\ell \in J$,

$$
\sum_{n \in \mathbb{Z}}\left|\hat{\phi}_{\ell}(\gamma+n / a)\right|^{2} \leq a B, \text { a.e. }
$$

Using estimates of this type for $\left\{T_{k a} \phi_{j}\right\}_{k \in \mathbb{Z}}$ as well as $\left\{T_{k a} \tilde{\phi}_{j}\right\}_{k \in \mathbb{Z}}$, we arrive at

$$
\begin{aligned}
& \sum_{j \in J} \sum_{n \in \mathbb{Z}}\left|\hat{\phi}_{j}(\gamma) \hat{\phi}_{\ell}(\gamma+n / a) \overline{\tilde{\tilde{\phi}}_{j}(\gamma+n / a)}\right| \\
& \quad \leq \sum_{j \in J}\left|\hat{\phi}_{j}(\gamma)\right|\left(\sum_{n \in \mathbb{Z}}\left|\hat{\phi}_{\ell}(\gamma+n / a)\right|^{2}\right)^{1 / 2}\left(\sum_{n \in \mathbb{Z}}\left|\hat{\tilde{\phi}}_{j}(\gamma+n / a)\right|^{2}\right)^{1 / 2} \\
& \quad \leq C \sum_{j \in J}\left|\hat{\phi}_{j}(\gamma)\right|
\end{aligned}
$$

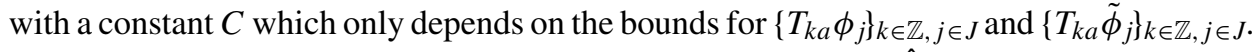
Thus, the series in (5.1) is unconditionally convergent if $\sum_{j \in J}\left|\hat{\phi}_{j}(\gamma)\right|<\infty$, a.e.

The example below shows that condition (5.3) is just a special case where condition (ii) in Corollary 3 is satisfied: 
Example 1. Let $\phi, \tilde{\phi} \in L^{2}(\mathbb{R})$, and take $a=1$. In the case of a single generator, (5.3) means that

$$
\hat{\phi}(\gamma) \overline{\tilde{\tilde{\phi}}(\gamma+n)}=\delta_{n, 0} \text { a.e. on }\left\{\gamma:|\hat{\phi}(\gamma)|^{2} \neq 0\right\} .
$$

On the other hand, condition (ii) in Corollary 3 means that

$$
\hat{\phi}(\gamma)=\sum_{n \in \mathbb{Z}} \hat{\phi}(\gamma) \overline{\tilde{\tilde{\phi}}(\gamma+n)} \hat{\phi}(\gamma+n),
$$

i.e., that

$$
\sum_{n \in \mathbb{Z}} \hat{\phi}(\gamma+n) \overline{\tilde{\tilde{\phi}}(\gamma+n)}=1 \text { a.e. on }\left\{\gamma: \sum_{n \in \mathbb{Z}}|\hat{\phi}(\gamma+n)|^{2} \neq 0\right\} \text {. }
$$

The condition (5.6) clearly does not force (5.5) to hold. However, let us give some concrete examples in terms of B-splines. Given $N$, let $B_{N}$ be the B-spline defined via $\hat{B_{N}}(\gamma)=$ $\left(\frac{\sin \pi \gamma}{\pi \gamma}\right)^{N}$. Let $M \in \mathbb{N}$ and define $\phi, \tilde{\phi}$ via $\phi=B_{N}$ and

$$
\hat{\tilde{\phi}}(\gamma)=\frac{\hat{B}_{N+2 M}(\gamma)}{\sum_{k \in \mathbb{Z}} \hat{B}_{N}(\gamma+k) \hat{B}_{N+2 M}(\gamma+k)} .
$$

Then condition (5.6) holds; and the expansion (5.2) of $\hat{\phi}$ in terms of translates $\hat{\phi}(\cdot+n), n \in$ $\mathbb{Z}$, is

$$
\begin{aligned}
\hat{\phi}(\gamma) & =\sum_{n \in \mathbb{Z}}(\hat{\phi}(\gamma) \hat{\tilde{\phi}}(\gamma+n)) \hat{\phi}(\gamma+n) \\
& =\sum_{n \in \mathbb{Z}}\left[\left(\frac{\sin \pi \gamma}{\pi \gamma}\right)^{N} \frac{\left(\frac{\sin \pi(\gamma+n)}{\pi(\gamma+n)}\right)^{N+2 M}}{\sum_{k \in \mathbb{Z}}\left(\frac{\sin \pi(\gamma+k)}{\pi(\gamma+k)}\right)^{2 N+2 M}}\right] \hat{\phi}(\gamma+n) .
\end{aligned}
$$

Clearly, in this case the condition in (5.5) does not hold.

\section{Gabor Systems}

Let $a, b>0$ be given. Our first purpose is to find conditions on $\phi, \tilde{\phi} \in L^{2}(\mathbb{R})$ such that the Gabor system $\left\{E_{j b} T_{k a} \tilde{\phi}\right\}_{j, k \in \mathbb{Z}}$ is a generalized dual of the Gabor system $\left\{E_{j b} T_{k a} \phi\right\}_{j, k \in \mathbb{Z}}$. To this end, we rely on the fact that $\left\{E_{j b} T_{k a} \tilde{\phi}\right\}$ and $\left\{E_{j b} T_{k a} \phi\right\}$ are generalized duals, if and only if the systems $\left\{T_{k a} E_{j b} \tilde{\phi}\right\}$ and $\left\{T_{k a} E_{j b} \phi\right\}$ are generalized duals. These systems have the structure of (2.1) with

$$
a_{j}=a, \phi_{j}=E_{j b} \phi, \tilde{\phi}_{j}=E_{j b} \tilde{\phi} .
$$

Corollary 4. Let $\phi, \tilde{\phi} \in L^{2}(\mathbb{R})$. Let $a, b>0$, assume that $\left\{E_{j b} T_{k a} \tilde{\phi}\right\}_{j, k \in \mathbb{Z}}$ and $\left\{E_{j b} T_{k a} \phi\right\}_{j, k \in \mathbb{Z}}$ are Bessel sequences, and that the series

$$
\sum_{j \in \mathbb{Z}} \sum_{n \in \mathbb{Z}} \hat{\phi}(\gamma+j b) \hat{\phi}(\gamma+n / a) \overline{\hat{\tilde{\phi}}(\gamma+n / a+j b)}
$$


converges unconditionally. Then the following are equivalent:

(i) $f=\sum_{j \in \mathbb{Z}} \sum_{k \in \mathbb{Z}}\left\langle f, E_{j b} T_{k a} \tilde{\phi}\right\rangle E_{j b} T_{k a} \phi, \forall f \in \overline{\operatorname{span}}\left\{E_{j b} T_{k a} \phi\right\}_{j, k \in \mathbb{Z}}$.

(ii) $\hat{\phi}(\gamma)=\sum_{n \in \mathbb{Z}}\left(\frac{1}{a} \sum_{j \in \mathbb{Z}} \hat{\phi}(\gamma+j b) \overline{\tilde{\tilde{\phi}}(\gamma+j b+n / a)}\right) \hat{\phi}(\gamma+n / a)$, a.e. $\gamma$.

In particular, the condition (ii) is satisfied, if

$$
\sum_{j \in \mathbb{Z}} \hat{\phi}(\gamma+j b) \overline{\tilde{\hat{\phi}}(\gamma+j b+n / a)}=a \delta_{n, 0} \text { a.e. on }\left\{\gamma: \sum_{j \in \mathbb{Z}}|\hat{\phi}(\gamma+j b)|^{2} \neq 0\right\} \text {. }
$$

Proof. Note that (i) holds, if and only if $\left\{T_{k a} E_{j b} \tilde{\phi}\right\}_{j, k \in \mathbb{Z}}$ is a generalized dual of $\left\{T_{k a} E_{j b} \phi\right\}_{j, k \in \mathbb{Z}}$; thus, we can apply Corollary 3 with

$$
\phi_{j}=E_{j b} \phi, \tilde{\phi}_{j}=E_{j b} \tilde{\phi} .
$$

Consider the following ordering of (5.1):

$$
\begin{aligned}
& \sum_{n \in \mathbb{Z}}\left(\sum_{j \in \mathbb{Z}} \hat{\phi}_{j}(\gamma) \overline{\hat{\tilde{\phi}}_{j}(\gamma+n / a)}\right) \hat{\phi}_{\ell}(\gamma+n / a) \\
& \quad=\sum_{n \in \mathbb{Z}}\left(\sum_{j \in \mathbb{Z}} \hat{\phi}(\gamma+j b) \overline{\hat{\tilde{\phi}}(\gamma+n / a+j b)}\right) \hat{\phi}(\gamma+n / a+\ell b)
\end{aligned}
$$

we have to check that this series converges unconditionally for a.e. $\gamma$. However, replacing $\gamma$

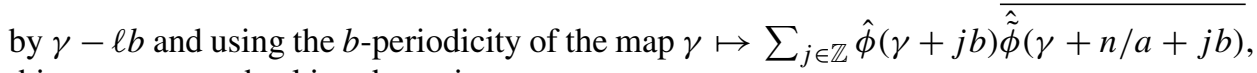
this amounts to checking the series

$$
\begin{aligned}
& \sum_{n \in \mathbb{Z}}\left(\sum_{j \in \mathbb{Z}} \hat{\phi}(\gamma-\ell b+j b) \overline{\hat{\tilde{\phi}}(\gamma-\ell b+n / a+j b)}\right) \hat{\phi}(\gamma+n / a) \\
& \quad=\sum_{n \in \mathbb{Z}}\left(\sum_{j \in \mathbb{Z}} \hat{\phi}(\gamma+j b) \overline{\tilde{\tilde{\phi}}(\gamma+n / a+j b)}\right) \hat{\phi}(\gamma+n / a)
\end{aligned}
$$

for unconditional convergence.

Condition (ii) in Corollary 3 takes the form

$\hat{\phi}(\gamma+\ell b)=\sum_{n \in \mathbb{Z}}\left(\frac{1}{a} \sum_{j \in \mathbb{Z}} \hat{\phi}(\gamma+j b) \overline{\tilde{\hat{\phi}}(\gamma+n / a+j b)}\right) \hat{\phi}(\gamma+n / a+\ell b)$, a.e. $\gamma$

again, using the periodicity of the sum over $j$ and replacing $\gamma$ by $\gamma-\ell b$, this condition corresponds to the condition in (ii).

By Lemma 3, the series in (6.1) is unconditionally convergent, if $\left\{E_{j b} T_{k a} \tilde{\phi}\right\}_{j, k \in \mathbb{Z}}$ and $\left\{E_{j b} T_{k a} \phi\right\}_{j, k \in \mathbb{Z}}$ are Bessel sequences and

$$
\sum_{j \in \mathbb{Z}}|\hat{\phi}(\gamma+j b)|<\infty \text { a.e. ; }
$$


the later condition is satisfied, e.g., if $\hat{\phi}$ belongs to the Wiener-type space

$$
W:=\left\{g: \mathbb{R} \rightarrow \mathbb{C}: g \text { is measurable and } \sum_{k \in \mathbb{Z}}\left\|g \chi_{[k, k+1[}\right\|_{\infty}<\infty\right\} .
$$

It is known that $\hat{\phi} \in W$, if $\phi$ belongs to Feichtingers algebra $\mathcal{S}_{0}$, cf. [10].

\section{Wavelet Systems in $L^{2}(\mathbb{R})$}

In this section we investigate the condition (4.4) for wavelet systems; in particular, we will prove that we can eliminate the dependence on the parameter $\ell$.

Let $\phi \in L^{2}(\mathbb{R})$ and consider the functions $\left\{2^{j / 2} \phi\left(2^{j} x-k\right)\right\}_{j, k \in \mathbb{Z}}$, i.e., the associated wavelet system. Letting $(D f)(x)=2^{1 / 2} f(2 x)$, the wavelet system can be written as $\left\{D^{j} T_{k} \phi\right\}_{j, k \in \mathbb{Z}}$. Via the commutator relations between the dilation operators and the translation operators, this system equals $\left\{T_{k 2^{-j}} D^{j} \phi\right\}_{j, k \in \mathbb{Z}}$; this system has the structure (2.1) of a generalized shift-invariant system with

$$
a_{j}=2^{-j}, \phi_{j}=D^{j} \phi
$$

Let

$$
\Lambda=\left\{2^{j} n: j \in \mathbb{Z}, n \in \mathbb{Z}\right\}
$$

and for $\alpha \in \Lambda$,

$$
J_{\alpha}=\left\{j \in \mathbb{Z}: \exists n \in \mathbb{Z}^{d} \text { such that } \alpha=2^{j} n\right\} .
$$

Using that $\mathcal{F} D^{j}=D^{-j} \mathcal{F}$, we can now formulate the condition (4.4) as

$$
\hat{\phi}\left(2^{-\ell} \gamma\right)=\sum_{\alpha \in \Lambda}\left(\sum_{j \in J_{\alpha}} \hat{\phi}\left(2^{-j} \gamma\right) \overline{\tilde{\tilde{\phi}}\left(2^{-j}(\gamma+\alpha)\right)}\right) e^{-2 \pi i 2^{-\ell} m \alpha} \hat{\phi}\left(2^{-\ell}(\gamma+\alpha)\right), \text { a.e. } \gamma
$$

This identity has to hold for all $\ell, m \in \mathbb{Z}$. Our goal is now to prove that it is enough to check the condition for $\ell=0$ and $m \in \mathbb{Z}$. We begin with a lemma, which follows immediately from the definition of $J_{\alpha}$.

Lemma 4. For $\alpha \in \Lambda$ and $\ell \in \mathbb{Z}$, we have $J_{2^{\ell} \alpha}=J_{\alpha}+\ell$.

Proposition 2. The condition (7.1) is satisfied for all $m, \ell \in \mathbb{Z}$, if and only if for all $m \in \mathbb{Z}$

$$
\hat{\phi}(\gamma)=\sum_{\alpha \in \Lambda}\left(\sum_{j \in J_{\alpha}} \hat{\phi}\left(2^{-j} \gamma\right) \overline{\tilde{\tilde{\phi}}\left(2^{-j}(\gamma+\alpha)\right)}\right) e^{-2 \pi i m \alpha} \hat{\phi}(\gamma+\alpha) \text {, a.e. } \gamma \text {. }
$$

In particular, this condition is satisfied, if

$$
\sum_{j \in J_{\alpha}} \hat{\phi}\left(2^{-j} \gamma\right) \overline{\tilde{\hat{\phi}}\left(2^{-j}(\gamma+\alpha)\right)}=\delta_{\alpha, 0} \text { a.e. on }\left\{\gamma: \sum_{j \in \mathbb{Z}}\left|\hat{\phi}\left(2^{-j} \gamma\right)\right|^{2} \neq 0\right\} \text {. }
$$


Proof. In order to rewrite (7.1), we notice that replacing $\gamma$ by $2^{\ell} \gamma$, this identity takes the form

$$
\begin{aligned}
\hat{\phi}(\gamma) & =\sum_{\alpha \in \Lambda}\left(\sum_{j \in J_{\alpha}} \hat{\phi}\left(2^{-(j-\ell)} \gamma\right) \overline{\tilde{\tilde{\phi}}\left(2^{-(j-\ell)}\left(\gamma+2^{-\ell} \alpha\right)\right)}\right) e^{-2 \pi i 2^{-\ell} m \alpha} \hat{\phi}\left(\gamma+2^{-\ell} \alpha\right) \\
& =\sum_{\alpha \in \Lambda}\left(\sum_{j \in J_{\alpha}-\ell} \hat{\phi}\left(2^{-j} \gamma\right) \overline{\tilde{\tilde{\phi}}\left(2^{-j}\left(\gamma+2^{-\ell} \alpha\right)\right)}\right) e^{-2 \pi i 2^{-\ell} m \alpha} \hat{\phi}\left(\gamma+2^{-\ell} \alpha\right) \\
& =\sum_{\alpha \in \Lambda}\left(\sum_{j \in J_{2}-\ell_{\alpha}} \hat{\phi}\left(2^{-j} \gamma\right) \overline{\tilde{\tilde{\phi}}\left(2^{-j}\left(\gamma+2^{-\ell} \alpha\right)\right)}\right) e^{-2 \pi i 2^{-\ell} m \alpha} \hat{\phi}\left(\gamma+2^{-\ell} \alpha\right) .
\end{aligned}
$$

For any $\ell \in \mathbb{Z}$, when $\alpha$ runs through $\Lambda, 2^{-\ell} \alpha$ runs through $\Lambda$ as well. We can therefore replace the sum over $\alpha$ with a sum over $2^{-\ell} \alpha$; this yields the conclusion. The second part follows directly from the last part of Corollary 1.

In order to check the conditions in Proposition 2 in practice, we need to calculate the functions $\Phi_{\alpha}, \alpha \in \Lambda$ defined by

$$
\Phi_{\alpha}(\gamma)=\sum_{j \in J_{\alpha}} \hat{\phi}\left(2^{-j} \gamma\right) \overline{\tilde{\tilde{\phi}}\left(2^{-j}(\gamma+\alpha)\right)}
$$

\section{Lemma 5.}

(i) If $\alpha \in \Lambda$ is an odd integer, then

$$
\Phi_{\alpha}(\gamma)=\sum_{j=0}^{\infty} \hat{\phi}\left(2^{j} \gamma\right) \overline{\tilde{\hat{\phi}}\left(2^{j}(\gamma+\alpha)\right)} .
$$

(ii) For a general $\alpha \in \Lambda \backslash\{0\}$, choose the odd integer $\alpha^{\prime}$ and $j^{\prime} \in \mathbb{Z}$ such that $\alpha=$ $2^{j^{\prime}} \alpha^{\prime}$. Then

$$
\Phi_{\alpha}(\gamma)=\Phi_{\alpha^{\prime}}\left(2^{-j^{\prime}} \gamma\right) .
$$

Proof. When $\alpha \in \Lambda$ is an odd integer, $J_{\alpha}=\{0,-1,-2, \ldots\}$; this immediately gives (i). For the proof of (ii), the choice of $\alpha^{\prime}, j^{\prime}$ implies by Lemma 4 that $J_{\alpha}=J_{\alpha^{\prime}}+j^{\prime}$. Thus,

$$
\begin{aligned}
\Phi_{\alpha}(\gamma) & =\sum_{j \in J_{\alpha}} \hat{\phi}\left(2^{-j} \gamma\right) \overline{\tilde{\tilde{\phi}}\left(2^{-j}\left(\gamma+2^{j^{\prime}} \alpha^{\prime}\right)\right)} \\
& =\sum_{j \in J_{\alpha^{\prime}}+j^{\prime}} \hat{\phi}\left(2^{-\left(j-j^{\prime}\right)} 2^{-j^{\prime}} \gamma\right) \overline{\tilde{\tilde{\phi}}\left(2^{-\left(j-j^{\prime}\right)}\left(2^{-j^{\prime}} \gamma+\alpha^{\prime}\right)\right)} \\
& =\sum_{j \in J_{\alpha^{\prime}}} \hat{\phi}\left(2^{-j} 2^{-j^{\prime}} \gamma\right) \overline{\tilde{\tilde{\phi}}\left(2^{-j}\left(2^{-j^{\prime}} \gamma+\alpha^{\prime}\right)\right)} \\
& =\Phi_{\alpha^{\prime}}\left(2^{-j^{\prime}} \gamma\right) .
\end{aligned}
$$

In particular, Lemma 5 shows that (7.1) is satisfied, if

$$
\sum_{j \in \mathbb{Z}} \hat{\phi}\left(2^{-j} \gamma\right) \overline{\hat{\tilde{\phi}}\left(2^{-j} \gamma\right)}=1 \text { and } \Phi_{\alpha}(\gamma)=0 \text { when } \alpha \text { is an odd integer ; }
$$


hereby we have recovered the conditions by Frazier et al. [8] for $\left\{D^{j} T_{k} \phi\right\}_{j, k \in \mathbb{Z}}$ and $\left\{D^{j} T_{k} \tilde{\phi}\right\}_{j, k \in \mathbb{Z}}$ being dual frames for $L^{2}(\mathbb{R})$, except that we did not prove the completeness in this special case.

\section{References}

[1] Bownik, M. and Weber, E. (2003). Affine frames, GMRA's and the canonical dual, Studia Math. 159(3), 453-479.

[2] Christensen, O. (2003). An Introduction to Frames and Riesz Bases, Birkhäuser.

[3] Christensen, O. and Eldar, Y. C. (2004). Oblique dual frames and shift-invariant spaces, Appl. Comp. Harm. Anal. 17(1), 48-68.

[4] Christensen, O., Deng, B., and Heil, C. (1999). Density of Gabor frames, Appl. Comp. Harm. Anal. 7, 292-304.

[5] Daubechies, I. (1992). Ten Lectures on Wavelets, CBMS-NSF Series in Appl. Math., SIAM, Philadelphia.

[6] Eldar, Y.C. (2003). Sampling with arbitrary sampling and reconstruction spaces and oblique dual frame vectors, J. Fourier Anal. Appl. 9(1), 77-96.

[7] Eldar, Y.C. (2003). Sampling without input constraints: Consistent reconstruction in arbitrary spaces, in Sampling, Wavelets and Tomography, Zayed, A. and Benedetto, J.J., Eds., Birkhäuser.

[8] Frazier, M., Garrigos, G., Wang, K. and Weiss, G. (1997). A characterization of functions that generate wavelet and related expansion, J. Fourier Anal. Appl. 3, Special Issue, 883-906.

[9] Gabardo, J.-P. and Han, D. (2004). The uniqueness of the dual of Weyl-Heisenberg subspace frames, Appl. Comp. Harm. Anal. 17(2), 226-240.

[10] Gröchenig, K. (2001). Foundations of Time-Frequency Analysis, Birkhäuser.

[11] Hernandez, E., Labate, D., and Weiss, G. (2002). A unified characterization of reproducing systems generated by a finite family II, J. Geom. Anal. 12(4), 615-662.

[12] Janssen, A. J.E. M. (1998). The duality condition for Weyl-Heisenberg frames, in Gabor Analysis: Theory and Application, Feichtinger, H.G. and Strohmer, T.. Eds., Birkhäuser.

[13] Janssen, A. J.E. M. (2001). Representations of Gabor frame operators, in Twentieth Century Harmonic Analysis - A Celebration, 73-101, NATO Sci. Ser. II, Math. Phys. Chem. 33, Kluwer Acad. Publ.

[14] Li, S. and Ogawa, H. (2004). Pseudoframes for subspaces with applications, J. Fourier Anal. Appl. 10(4), 409-431.

[15] Ron, A. and Shen, Z. (1995). Frames and stable bases for shift-invariant subspaces of $L^{2}\left(\mathbb{R}^{d}\right)$, Canad. J. Math. 47(5), 1051-1094.

[16] Ron, A. and Shen, Z. (2004). Generalized shift-invariant systems, Const. Appr. to appear.

[17] Werther, T. and Eldar, Y.C. (2005). General framework for consistent sampling in Hilbert spaces, Int. J. Wavelets Multiresolut. Inf. Process. to appear.

[18] Wexler, J. and Raz, S. (1990). Discrete Gabor expansions, Signal Processing 21, 207-220.

Received May 10, 2004

Revision received January 26, 2005

Department of Mathematics, Technical University of Denmark, Building 3032800 Lyngby, Denmark e-mail: Ole.Christensen@mat.dtu.dk

Technion - Israel Institute of Technology, Department of Electrical Engineering,

Technion City, Haifa, 32000, Israel

e-mail: yonina@ee.technion.ac.il 